\title{
"The Immune Conundrum": Acquired Hemophilia A, Immune Thrombocytopenia, and Neutropenia in a Patient with Pancreatic Cancer
}

\author{
Praveen Ramakrishnan Geethakumari, ${ }^{1}$ Ashwin Sama, ${ }^{2}$ Jaime G. Caro, ${ }^{3}$ Charles J. Yeo, ${ }^{4}$ and Srikanth Nagalla ${ }^{3}$
}

\begin{abstract}
Background: Malignancy-associated bleeding can pose diagnostic dilemmas. We report a unique case of paraneoplastic acquired hemophilia A (AHA), immune thrombocytopenia (ITP), and immune neutropenia in a patient with pancreatic adenocarcinoma.

Case Presentation: A 66-year-old male with newly diagnosed pancreatic cancer and normal preoperative hematological evaluation was taken to the operating room for pancreaticoduodenectomy. The operation was aborted due to empyema of the gall bladder, cholangitis, and local extent of disease. Postoperatively, the patient developed bleeding diatheses with mucocutaneous and intra-abdominal bleeding and a prolonged activated partial thromboplastin time. Evaluation revealed high-titer factor VIII inhibitor confirming AHA. Management with bypassing agents such as recombinant activated factor VII, factor VIII inhibitor bypassing activity, and immunosuppression with steroids, cyclophosphamide, and rituximab achieved remission in 2 months. ITP developed after achieving normal factor VIII levels, which was managed with intravenous immunoglobulin. Neutropenia was detected before initiation of chemotherapy and was managed with granulocyte-colony stimulating factor. Conclusion: These unique challenges posed by paraneoplastic hematological syndromes warrant the need for astute clinical judgment and multidisciplinary collaboration for effective management.
\end{abstract}

Key words: acquired hemophilia A; immune thrombocytopenia; neutropenia; pancreatic cancer; paraneoplastic syndromes

\section{Introduction}

Malignancy-associated bleeding can be multifactorial posing diagnostic dilemmas. ${ }^{1}$ Acquired hemophilia A (AHA) is a rare life-threatening disorder caused by autoantibodies to factor VIII that may occur spontaneously (50\% of cases) or secondary to malignancy, pregnancy, autoimmune disease, or medications. ${ }^{2,3}$ Autoimmune thrombocytopenia and neutropenia are uncommon paraneoplastic syndromes. ${ }^{4}$

We describe a challenging presentation of acquired immune dysregulation causing three distinct autoimmune syndromes of AHA, immune thrombocytopenia (ITP), and immune neutropenia.

\section{Case Report}

A 66-year-old Caucasian male presented with obstructive jaundice in January 2015. He was found to have a hypodense mass in the head of pancreas and underwent biliary stent placement. Endoscopic ultrasound and fine needle aspiration revealed adenocarcinoma of the pancreas. The tumor was staged as IB, cT2cN0cM0. The patient was scheduled for a Whipple procedure at the end of February. At surgical exploration, empyema of the gall bladder, pyobilia, and locally advanced tumor were discovered. Surgical resection was aborted and Roux-en-Y hepaticojejunostomy was performed for optimal biliary drainage. No excessive bleeding

Departments of ${ }^{1}$ Hematology and Medical Oncology, ${ }^{2}$ Medical Oncology, ${ }^{3}$ Hematology and ${ }^{4}$ Department of Surgery, Thomas Jefferson University Hospital, Philadelphia, Pennsylvania.

*Address correspondence to: Praveen Ramakrishnan Geethakumari, MD, Department of Hematology and Medical Oncology, 834 Chestnut Street, Suite 320 , Thomas Jefferson University Hospital, Philadelphia, PA 19107, E-mail: praveen.ramakrishnan@jefferson.edu

(C) Praveen Ramakrishnan Geethakumari et al. 2016; Published by Mary Ann Liebert, Inc. This Open Access article is distributed under the terms of the Creative Commons License (http://creativecommons.org/licenses/by/4.0), which permits unrestricted use, distribution, and reproduction in any medium, provided the original work is properly credited. 
was encountered during surgery. Broad spectrum antibiotics were started intraoperatively and continued perioperatively.

Preoperative hematological evaluation was within normal limits. Postoperatively, the patient developed bleeding from drains and intravenous line sites. Prothrombin time was mildly increased at $13.9 \mathrm{sec}$ and activated partial thromboplastin time (aPTT) was elevated at $85 \mathrm{sec}$. A 1:1 aPTT mixing study did not correct, suggesting the presence of an inhibitor. Further analysis showed a factor VIII level of $1 \%$ and factor VIII inhibitor titer of 14.3 Bethesda units (BU). Specific tests such as lupus anticoagulant, dilute Russel viper venom test, thrombin time, and other coagulation factors were within normal limits (Fig. 1 and Table 1).

The patient developed abdominal wall ecchymoses, and multiple intra-abdominal hematomas were detected on axial CT imaging. His hemoglobin dropped from 13.7 to $7.4 \mathrm{~g} / \mathrm{dL}$ and was supported with packed red blood cell (PRBC) transfusions. He was immediately started on recombinant activated factor VII (rFVIIa) and intravenous immunoglobulin (IVIG) therapies, which controlled bleeding but inhibitor titer increased to $37 \mathrm{BU}$. Cyclophosphamide was initiated at a dose of $2 \mathrm{mg} / \mathrm{kg}$ body weight $(200 \mathrm{mg})$ daily. The inhibitor titer peaked at $243.9 \mathrm{BU}$ and factor VIII at nadir was $<1 \%$ (Fig. 1). He required 6 units of PRBCs. The patient was discharged on a regimen of prednisone and cyclophosphamide.

He was readmitted for gastrointestinal bleeding and symptomatic anemia. Factor VIII was still $<1 \%$ and the inhibitor titer was $142.9 \mathrm{BU}$. A descending colonic source of presumed diverticular bleeding was identified and managed with PRBC transfusions and restarting rfVIIa. No interventional procedure was performed due to the risk of catastrophic bleeding. Rituximab was initiated at $375 \mathrm{mg} / \mathrm{m}^{2}$ weekly for four doses. Factor VIII inhibitor bypassing activity (FEIBA) was then started due to the frequent need of transfusions. He required 13 units of PRBCs. He was discharged on a regimen of rfVIIa, cyclophosphamide, prednisone, and completed four doses of rituximab. The factor VIII levels improved to $13 \%$ and inhibitor titer decremented to $<0.6 \mathrm{BU}$ (Fig. 1).

Moderate thrombocytopenia was detected on follow-up with platelet count of $35,000 / \mu \mathrm{L}$. The cyclophosphamide dose was decreased to $150 \mathrm{mg}$ daily. The platelet count dropped further to $4000 / \mu \mathrm{L}$ and the patient developed wet purpura in the oral cavity. As RBC and WBC counts were stable, he was initiated on IVIG for ITP, resulting in normalization of platelet count
(Fig. 1). He was discharged on a regimen of cyclophosphamide and slow steroid taper.

Repeat laboratories showed a decline in WBC count to $200 / \mu \mathrm{L}$, with a normal platelet count and hemoglobin. This was diagnosed as autoimmune neutropenia. The steroid dose was increased to $60 \mathrm{mg}$ daily, cyclophosphamide stopped, and granulocyte-colony stimulating factor (G-CSF) initiated (daily for four doses), with prompt recovery of WBC counts (Fig. 1).

He was evaluated by a multidisciplinary team of hematology, and medical, radiation, and surgical oncology. After blood count recovery, a short course of radiation therapy was started for pancreatic cancer followed by chemotherapy.

\section{Discussion and Literature Review}

Paraneoplastic hematological syndromes include AHA, ITP, autoimmune hemolytic anemia, antiphospholipid antibodies, granulocytosis, eosinophilia, thrombocytosis, anemia, and pure red cell aplasia. ${ }^{4}$ We are reporting the first patient, to the best of our knowledge, to develop AHA, ITP, and autoimmune neutropenia in a postoperative setting for pancreatic cancer. The other unique feature is the development of autoimmune cytopenias after the patient achieved normal factor VIII levels on multiple immunosuppressants.

The postulated mechanism of AHA is immune dysfunction from an abnormal T-cell response to a stimulatory antigen or abnormal interactions between $\mathrm{T}$ and $\mathrm{B}$ cells leading to autoantibody generation. Research has shown variations in the CTLA-4 gene and upregulation of $\mathrm{B}$-cell activating factor in cases of AHA. Acquired factor VIII inhibitors exhibit type 2 kinetics with rapid initial inactivation of factor VIII followed by a slower phase of equilibrium, compared to alloantibodies in congenital hemophilia A that follow type I kinetics. ${ }^{2,5}$

AHA accounts for high-mortality rates ranging between $7 \%$ and $22 \% .{ }^{6}$ Severe bleeding (spontaneous [75\%] and provoked [25\%]) and unexplained prolonged aPTT are the most common presentations. Spontaneous subcutaneous, muscular, and retroperitoneal bleeds are common, but mucosal and intracranial bleeds have been reported. Hemarthrosis is uncommon unlike congenital hemophilia. About 5-7\% patients can present with isolated laboratory abnormalities. ${ }^{2,3}$

AHA can be associated with underlying malignancy in $6.7-14 \%$ patients. The prevalence of paraneoplastic factor VIII inhibitors was reported to be $11.8 \%$ from 

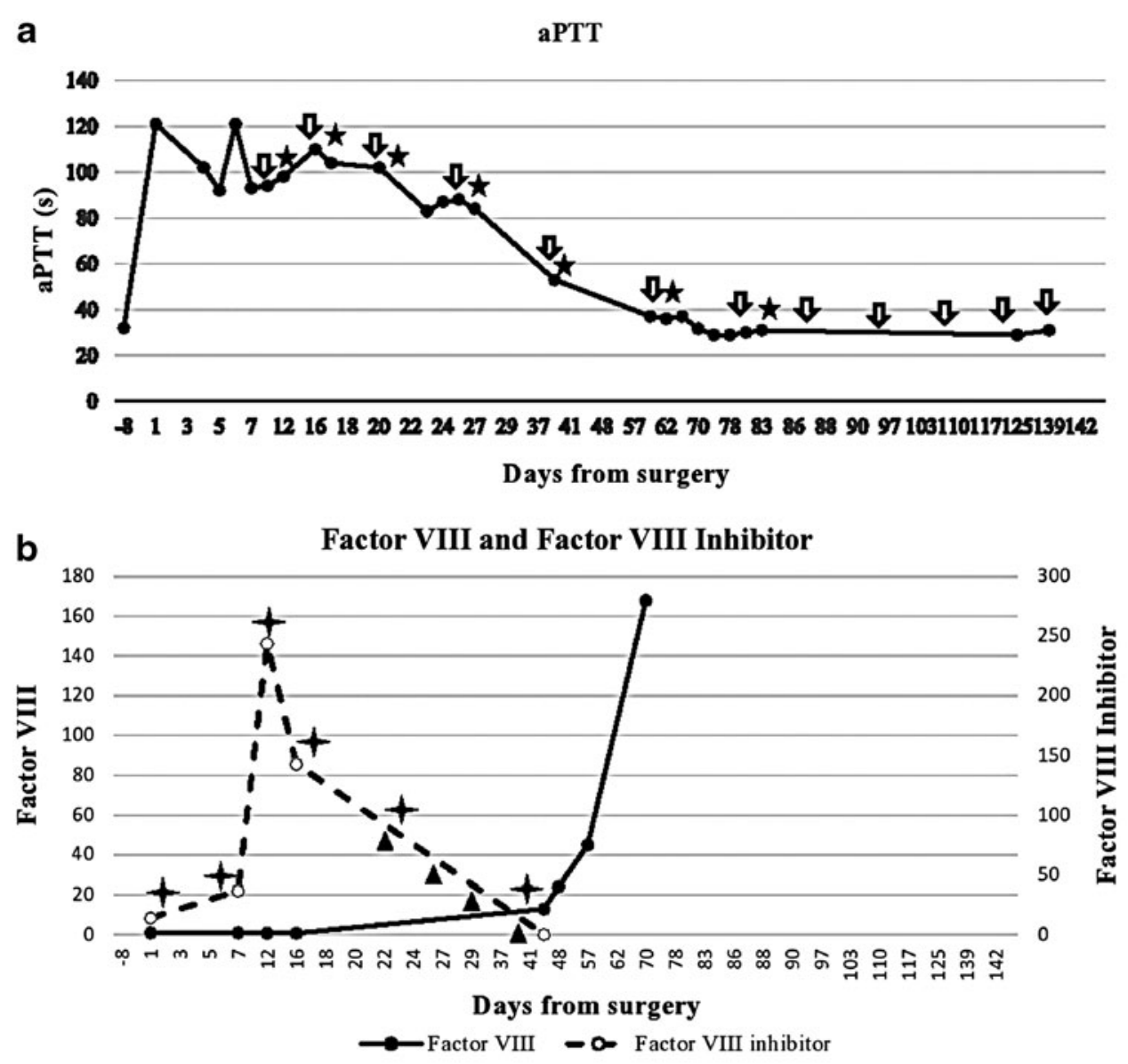

C

\section{Platelets and ANC}

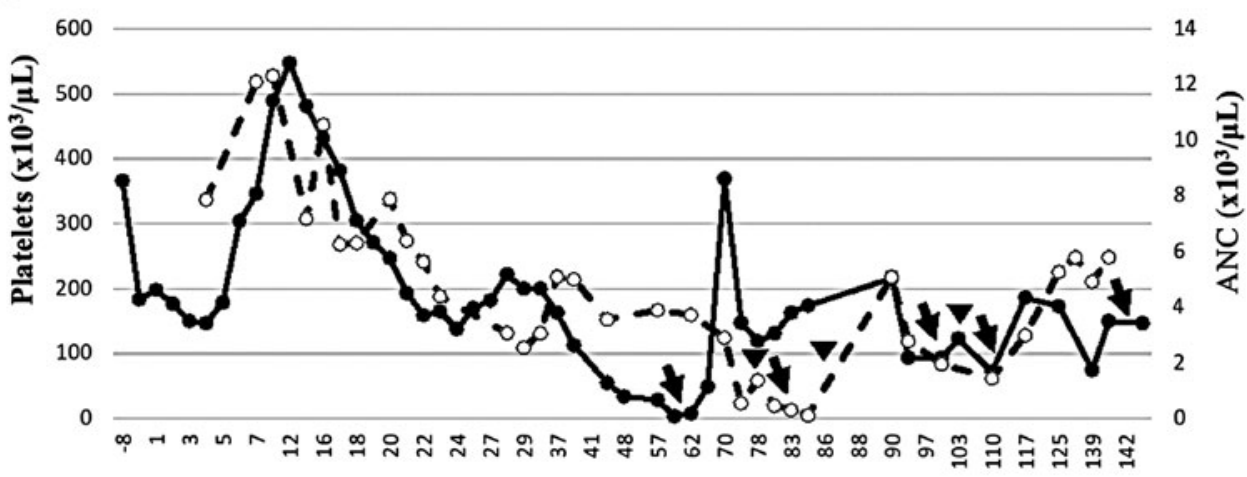

Days from surgery

$\rightarrow$ Platelets $\quad \infty$ ANC

\section{VPrednisone $\star$ Cyclophosphamide + rfVIIa $\Delta$ Rituximab \ IVIG $\nabla$ G-CSF}

FIG. 1. Trends of (a) activated partial thromboplastin time (aPTT), (b) factor VIII level and inhibitor titer, (c) platelet and absolute neutrophil count (ANC) and therapeutic interventions. 
Table 1. Normal Laboratory Values Compared to Immediate Post-Operative Values of Patient

Normal laboratory values (general population)

Hemoglobin, $\mathrm{g} / \mathrm{dL} \quad 14-17$

White blood cell count, $\times 10^{9} / \mathrm{L}$

Absolute neutrophil, $\times 10^{9} / \mathrm{L} \quad 1.6-8$

Platelet count, $\times 10^{9} / \mathrm{L} \quad 140-400$

Prothrombin time, sec $\quad 8.9-13.3$

aPTT, sec 26-38

Factor VIII activity, \% 41-159

Immediate postoperative laboratory values of patient (Post-op Day 0 )

aPTT, sec, $N=26-38$

121

aPTT mix, sec

52.4

Factor VIII activity, \%, N=41-159

Factor IX activity, \%, N=50-160

Factor Xl activity, \%, $N=58-143$

Factor XIl activity, \%, N=48-158

Thrombin time, sec, $N=11.2-17.3$

dRVVT, sec, $N=24.4-37.2$

1

97

74

59

12.4

Factor VIII inhibitor, $\mathrm{BU}, \mathrm{N}=<0.6 \mathrm{BU} / \mathrm{mL}$

35.1

14.3

aPTT, activated partial thromboplastin time; BU, Bethesda units; dRVVT, dilute Russel viper venom test.

the European Acquired Hemophilia Registry (EACH2), $67.8 \%$ with solid tumors, and $32.2 \%$ with hematological malignancies. Among solid tumors, AHA has been reported more often in prostate, lung, and gastrointestinal cancers. ${ }^{2,7,8}$

About $20 \%$ of patients with paraneoplastic factor VIII antibodies develop these in conjunction with surgery for cancer. The majority of patients had gastrointestinal cancers and median age was 69 years. $^{9-13}$ They were predominantly male, in contrast to female predominance in nonsurgical paraneoplastic factor VIII inhibitors from the $\mathrm{EACH} 2$ registry. Triggering of paraneoplastic AHA by surgery is unique and has only been observed in syndromes like autoimmune hemolytic anemia. The median duration from surgery to antibody detection was 3 months (range 1 week to 6 months). Most patients had low antibody titers (median $14 \mathrm{BU}$; range $1.7-64 \mathrm{BU}) .{ }^{14}$ Our case is unique in the rapid occurrence of high-titer autoantibody to factor VIII following surgery. It is also possible that underlying pancreatic cancer, ${ }^{15}$ infection (cholangitis), or medications such as antibiotics and anesthetics during surgery triggered the abnormal antibody production.

Management of AHA is two pronged: (1) achievement of hemostasis and (2) eradication of the autoantibody production. ${ }^{16}$ Bypassing agents in the form of rFVIIa and FEIBA are the main treatment options for patients with major bleeding and high-titer inhibitors. Either agent has greater than $90 \%$ success rate when used as first-line therapy in AHA. If one agent fails to achieve adequate hemostasis, the other should be tried as in our case.

Immunosuppression to achieve inhibitor eradication can be complicated by the underlying infection as in our case. Current therapy involves the combination of steroids and cyclophosphamide that have success rates of up to $80 \%$. Rituximab is increasingly used as firstand second-line strategies and showed a 59\% complete remission $(\mathrm{CR})$ rate as single agent in the $\mathrm{EACH} 2$ registry. Other strategies include azathioprine, mycophenolate mofetil, and calcineurin inhibitors. ${ }^{17,18}$

Our patient needed bypassing agents, IVIG, steroids, cyclophosphamide, and rituximab to achieve hemostasis and inhibitor eradication. Sequential and combined use of these agents must be tailored to the patient's presentation. Disease flares on immunosuppression withdrawal may necessitate reintroduction of these agents.

The major cause of cancer-associated thrombocytopenia is secondary to bone marrow aplasia from chemotherapy or radiation. Less common causes are marrow infiltration by tumor, disseminated intravascular coagulation, and related to drug. Paraneoplastic ITP has been associated with hematological malignancies and solid tumors. In a review of 68 cases of paraneoplastic ITP with solid tumors, it was commonly described in tumors of the lung, breast, kidney, and ovary, and rarely in prostate cancer. It has only been described in one case of pancreatic cancer before this report. ${ }^{19}$ ITP can occur concurrently with the cancer diagnosis (50\%), preceding diagnosis (25\%), and the rest manifest during disease course or at recurrence. Most patients responded to steroid treatment or splenectomy and only few had a CR after definitive surgical resection or chemotherapy for the cancer [4]. Our patient had already received steroids, rituximab, and was on cyclophosphamide in remission from AHA, before ITP developed and had good response to IVIG. It is interesting to observe the emergence of ITP on immunosuppression.

The neutropenia before chemotherapy initiation may be attributed to the cytotoxic effect of cyclophosphamide, but with normal platelet count, improving hemoglobin, and appropriate reticulocyte count, paraneoplastic autoimmune neutropenia seems the more likely diagnosis. The patient responded to G-CSF, and reinitiation of cyclophosphamide did not affect the WBC count. The treatment sequence for pancreatic cancer was modified in our case to start with radiation therapy, followed by chemotherapy once platelet and neutrophil counts stabilized. 


\section{Conclusions}

This case describes a unique paraneoplastic immune conundrum manifesting as three entities of AHA, ITP, and neutropenia in pancreatic cancer. This reiterates the need for astute clinical judgment and multidisciplinary collaboration for making accurate diagnoses and instituting prompt therapy for patients. Research in cancer immunology should better elucidate paraneoplastic phenomena improving future treatment options.

\section{Acknowledgment}

The authors thank Ms. Rasmi Nair, MPH, for providing assistance for graphical representation of the data.

\section{Author Disclosure Statement}

No competing financial interests exist.

\section{References}

1. Escobar MA. Bleeding in the patient with a malignancy: is it an acquired factor VIII inhibitor? Cancer. 2012;118:312-320.

2. Franchini M, Lippi G. Acquired factor VIII inhibitors. Blood. 2008;112: 250-255.

3. Ma AD, Carrizosa D. Acquired factor VIII inhibitors: pathophysiology and treatment. Hematology Am Soc Hematol Educ Program. 2006:432-437.

4. Krauth MT, Puthenparambil J, Lechner K. Paraneoplastic autoimmune thrombocytopenia in solid tumors. Crit Rev Oncol Hematol. 2012;81: 75-81.

5. Sakurai Y, Takeda T. Acquired hemophilia A: a frequently overlooked autoimmune hemorrhagic disorder. J Immunol Res. 2014;2014:320674.

6. Aouba A, Rey G, Pavillon G, et al. Deaths associated with acquired haemophilia in France from 2000 to 2009: multiple cause analysis for best care strategies. Haemophilia. 2012;18:339-344.

7. Franchini $M$, Targher $G$, Manzato $F$, et al. Acquired factor VIII inhibitors in oncohematology: a systematic review. Crit Rev Oncol Hematol. 2008;66:194-199.

8. Sallah S, Wan JY. Inhibitors against factor VIII in patients with cancer. Analysis of 41 patients. Cancer. 2001;91:1067-1074.

9. Celestino F, Verri C, De Carlo F, et al. Localised prostate cancer and hemophilia A (AHA): case report and management of the disease. Arch Ital Urol Androl. 2014;86:227-228.

10. Hosoya Y, Matsumura M, Madoiwa S, et al. Acquired hemophilia A caused by factor VIII inhibitors: report of a case. Surg Today. 2013;43:670-674.

11. Mekenkamp LJ, Beishuizen A, Slomp J, et al. Successful treatment of fulminant postoperative bleeding due to acquired haemophilia. Neth J Med. 2015;73:182-186.
12. Miura T, Ban D, Koyama T, et al. Severe postoperative hemorrhage caused by antibody-mediated coagulation factor deficiencies: report of two cases. Surg Today. 2014;44:976-981.

13. Onishi I, Kayahara M, Munemoto $M$, et al. Management of postoperative hemorrhage associated with factor VIII inhibitor: report of a case. Surg Today. 2013;43:1058-1061.

14. Reitter S, Knoebl P, Pabinger I, et al. Postoperative paraneoplastic factor VIII auto-antibodies in patients with solid tumours. Haemophilia. 2011;17:e889-e894.

15. Rhodes EG, Boesen EA, Corringham RE, et al. Disappearance of antibodies to Factor VIII in a patient with acquired haemophilia and carcinoma of the pancreas during cytotoxic therapy with fluorouracil and CCNU. Thromb Haemost. 1984;52:131-133.

16. Sborov DW, Rodgers GM. How I manage patients with acquired haemophilia A. Br J Haematol. 2013;161:157-165.

17. Janbain M, Leissinger CA, Kruse-Jarres R. Acquired hemophilia A: emerging treatment options. J Blood Med. 2015;6:143-150.

18. Muzaffar J, Katragadda L, Haider S, et al. Rituximab and intravenous immunoglobulin (IVIG) for the management of acquired factor VIII inhibitor in multiple myeloma: case report and review of literature. Int J Hematol. 2012;95:102-106.

19. Bir A, Bshara W, George M, et al. Idiopathic thrombocytopenic purpura in a newly diagnosed pancreatic adenocarcinoma. JOP. 2006;7:647-650.

Cite this article as: Geethakumari PR, Sama A, Caro JG, Yeo CJ Nagalla S (2016) "The Immune Conundrum": acquired hemophilia A, immune thrombocytopenia, and neutropenia in a patient with pancreatic cancer, Case Reports in Pancreatic Cancer 2:1, 14-18, DOI: 10.1089/crpc.2015.29011.prg.

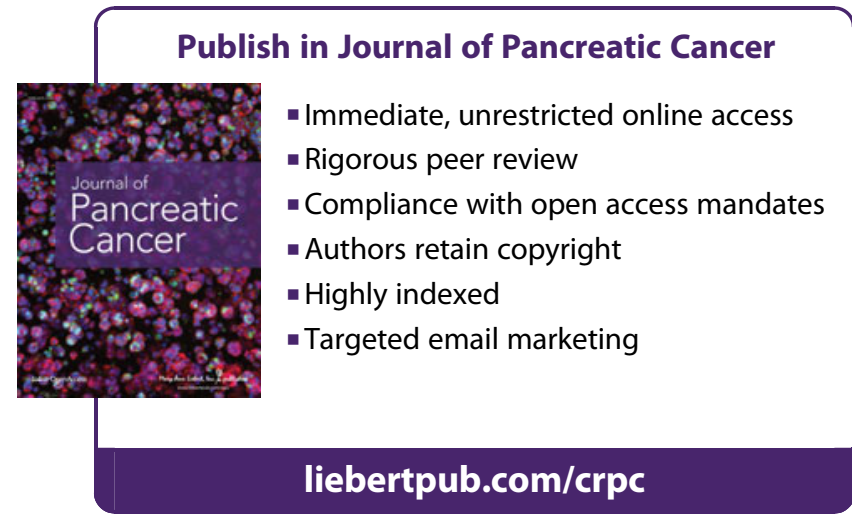

